\title{
Signal Space Diversity for Spatial Multiplexing
}

\author{
Gajanana Krishna, K. V. Srinivas, Srikrishna Bhashyam and R. D. Koilpillai \\ Department of Electrical Engineering \\ Indian Institute of Technology-Madras \\ Chennai-600036, India \\ gajananagk@iitm.ac.in, $\{$ kvsri,skrishna,koilpillai\}@ee.iitm.ac.in
}

\begin{abstract}
When channel state information is available at the transmitter, we can diagonalize a MIMO channel with SVD transceivers. However, outage of the weaker eigenchannels limits the performance of such a transceiver. In the current work, we propose and analytically characterize co-ordinate interleaving of multi-dimensional symbols over sets of eigenchannels. We show that in our method, the diversity multiplexing trade-off (DMT) is determined by the strongest eigenchannel in each set. We also calculate the optimal (DMT) for different possible sets and constellation dimensions with rate allocation among the sets.
\end{abstract}

\section{INTRODUCTION}

In slow fading delay limited channels, diversity multiplexing trade-off (DMT) [1] is a good measure of performance for any multiple antenna (MIMO) transmission scheme at high SNR. While [1] analyzed the optimum DMT with no channel state information at the transmitter (CSIT), a variety of methods have been proposed to improve the DMT with CSIT.

Diagonalizing a MIMO channel with singular value decomposition (SVD), is a well known technique when CSIT is available. The bank of parallel channels with channel gains given by the ordered singular values of the MIMO channel matrix are commonly known as eigenchannels or eigenmodes. In [2], multiple data streams are transmitted in parallel on these eigenchannels in what is called multiple beamforming. However, the DMT derived in [2] is lower than the DMT in [1] even with the advantage of CSIT. On account of the scalar encoding restriction, the method in [2] experiences outage when any of the eigenchannels in use experiences outage. The weaker eigenchannels, tend to be in outage more frequently than the stronger ones. As we increase the spatial multiplexing gain, the weaker eigenchannels have to be employed and there is a degradation of diversity compared to [1].

To solve the problem, interleaving the co-ordinates of twodimensional QAM symbols across eigenchannels was first proposed in [3]. Building on [3], in the current work, we divide the eigenchannels into sets and in each set we interleave the co-ordinates of multi-dimensional symbols across the different eigenchannels. Use of rotated multi-dimensional constellations to exploit signal space diversity was first proposed in [4] and analyzed in [5]. In our method, the different co-ordinates of the multi-dimensional symbols drawn from a rotated constellation pass through the different eigenchannels.

We characterize the symbol error rate with the diversitymultiplexing trade-off (DMT) [1] and show that with coordinate interleaving, the DMT is determined only by the statistics of the strongest eigenchannel in the set. Thus, coordinate interleaving is a technique where the weaker channels are hidden by the presence of a strong channel in the set. To the end of obtaining the DMT for the technique of coordinate interleaving, we obtain a novel approximation, for small arguments, to the joint probability distribution of any subset of the eigenvalues of a Wishart matrix.

With respect to the DMT, our method outperforms all other methods of beamforming operating under a short term power and rate constraint. The benefits can be ascribed to signal space diversity. We effectively employ joint vector coding across the eigenchannels as opposed to scalar encoding in models like [2]. When the dimension of the signal space is equal to the number of eigenchannels and the co-ordinates of the symbols are interleaved across all the eigenchannels, we get the best performance and the DMT is simply a straight line joining the points of maximum diversity and maximum spatial multiplexing. Noting that the decoding complexity grows with the dimension of the constellation, we propose appropriate partitioning of the eigenchannels into sets and optimum rate allocation across the different sets. Thus, the user can trade the performance off for decoding complexity by employing constellations with smaller dimensions. From our results, it follows that we get the most significant gains in the DMT at high multiplexing gains i.e. when the spatial degrees of freedom offered by the MIMO channel are being utilized.

The remainder of the paper is organized as follows. In section II, we present the system model and the technique of generalized co-ordinate interleaved spatial multiplexing. In section III, we analyze the symbol error rate (SER) and outage probability for our system. We conclude in Section V.

\section{System Model AND COORDINATE INTERLEAVING}

Consider a MIMO system with $N_{t}$ transmit antennas and $N_{r}$ receive antennas $\left(N_{t} \times N_{r}\right.$ system) where the channel experiences flat Rayleigh fading and additive white Gaussian noise. The discrete time baseband input-output relation of the MIMO channel is,

$$
\mathbf{y}=\mathbf{H s}+\mathbf{w},
$$

where $\mathbf{s} \in \mathbb{C}^{N_{t} \times 1}$ is the complex, information bearing symbol vector, $\mathbf{y} \in \mathbb{C}^{N_{r} \times 1}$ is the channel output vector, $\mathbf{H}=\left[h_{i j}\right] \in$ $\mathbb{C}^{N_{r} \times N_{t}}$ is the channel realization (normalized to unity gain) and $\mathbf{w} \in \mathbb{C}^{N_{r} \times 1}$ is the zero mean, circularly symmetric, complex Gaussian unit variance additive noise. We define $m=\max \left\{N_{t}, N_{r}\right\}, n=\min \left\{N_{t}, N_{r}\right\}$. 
We assume pefect channel state information at the receiver and transmitter. The MIMO channel is diagonalized through SVD as $\mathbf{H}=\mathbf{U} \boldsymbol{\Lambda} \mathbf{V}^{H}$ where $\mathbf{U} \in \mathbb{C}^{N_{r} \times n}$ and $\mathbf{V} \in \mathbb{C}^{N_{t} \times n}$ are unitary matrices and $\Lambda \in \mathbb{R}^{n \times n}$ is a diagonal matrix with $\sqrt{\lambda_{\ell}} \in \mathbb{R}^{+}$as its $\ell^{t h}$ diagonal element representing the $\ell^{t h}$ eigenchannel. Here $\sqrt{\lambda_{\ell}}$ denotes the gain of the $\ell^{t h}$ largest singular value of $\mathbf{H}$.

Our system operates under a short term power constraint $\mathbb{E}\left[\mathbf{s}^{\dagger} \mathbf{s}\right] \leq P$. We generalize the scheme in [3] for significantly improved performance with arbitrary $n$ using multidimensional QAM constellations, which were first proposed in [5] as a method of improving throughput at the expense of decoding complexity. We partition the $n$ eigenchannels into sets of eigenchannels $Q_{1}, Q_{2}, \ldots, Q_{s}$ such that $\sum_{i=1}^{s} q_{i}=n$ where $q_{i}=\left|Q_{i}\right|$, the number of elements in $Q_{i}$. Depending on the target data rate, we pick the first $L \leq s$ sets among $\left\{Q_{i}\right\}$ and allocate power $P_{i}$ to each set such that $\sum_{i=1}^{L} P_{i} \leq P$. We then use $q_{i}$-dimensional co-ordinate interleaved coding on each set $Q_{i}$ for $1 \leq i \leq L$.

Let $\mathbf{x}^{i}=\mathbf{x}_{1}^{i}+\jmath \mathbf{x}_{2}^{i}$ be the codeword on a particular set of eigenchannels $Q_{i}$ where $\jmath=\sqrt{-1}$. We choose $\mathbf{x}_{k}^{i}=\mathbf{M b}$ for $k=1,2$ where $\mathbf{b}=\left(b_{1}, b_{2}, \ldots, b_{q_{i}}\right)^{T}$ represents a point from $\mathcal{O}$, an $q_{i}$-dimensional QAM constellation and $\mathbf{M}=\left[m_{i j}\right] \in$ $\mathbb{R}^{q_{i} \times q_{i}}$ is the rotation matrix applied to the constellation to get $\mathcal{O}^{\prime}$ [5]. Let $\mathbf{s}^{i}=\mathbf{V}^{i} \overline{\mathbf{P}}^{i} \mathbf{x}_{i}$ where $\mathbf{V}^{i}$ contains the right singular vectors corresponding to the eigenchannels in $Q_{i}$ and $\overline{\mathbf{P}}^{i}=\operatorname{diag}\left(\left\{\sqrt{p_{k}^{i}}\right\}_{k=1, \ldots, q_{i}}\right)$ such that $\sum_{k=1}^{q_{i}} p_{k}^{i} \leq P_{i}$ the power constraint for $Q_{i}$. Transmit $\mathbf{s}=\sum_{i=1}^{L} \mathbf{s}^{i} \in \mathbb{C}^{N_{t} \times 1}$.

Multiply the received vector $\mathbf{y}$ by $\mathbf{U}^{H}$ to obtain the equivalent received signal on the set $Q_{i}$ as

$$
\mathbf{r}^{i}=\mathbf{r}_{1}^{i}+\jmath \mathbf{r}_{2}^{i}=\left(\boldsymbol{\Lambda}^{i} \overline{\mathbf{P}}^{i} \mathbf{x}_{1}^{i}+\mathbf{w}_{1}^{\prime}\right)+\jmath\left(\boldsymbol{\Lambda}^{i} \overline{\mathbf{P}}^{i} \mathbf{x}_{2}^{i}+\mathbf{w}_{2}^{\prime}\right),
$$

where $\boldsymbol{\Lambda}^{i}=\operatorname{diag}\left(\left\{\sqrt{\lambda_{\ell_{k}}}\right\}, k=1,2, \ldots, q_{i}\right)$ contains the eigenchannels in $Q_{i}$ and $\mathbf{w}_{1}^{\prime}=\Re \mathbf{w}^{\prime}, \mathbf{w}_{2}^{\prime}=\Im \mathbf{w}^{\prime}$ represent the noise vectors. ${ }^{1}$. If $\mathbf{x}_{t}^{i}=\left[x_{t 1}^{i} x_{t 2}^{i} \cdots x_{t q_{i}}^{i}\right]^{T}$ and $\mathbf{w}_{t}^{\prime}=\left[w_{t 1}^{\prime} w_{t 2}^{\prime} \cdots w_{t q_{i}}^{\prime}\right]^{T}$, the individual entries of $\mathbf{r}_{t}^{i}=$ $\left[r_{t 1}^{i} r_{t 2}^{i} \cdots r_{t q_{i}}^{i}\right]^{T}$ are given by

$$
r_{t k}^{i}=\sqrt{\lambda_{\ell_{j}} p_{k}^{i}} x_{t k}^{i}+w_{t k}^{\prime},
$$

for $t=1,2$. From (3), we see that the coordinates of the multidimensional symbols pass through different eigenchannels. The maximum-likelihood (ML) detection criterion with perfect CSI stipulates the minimization of the following metric to estimate the transmitted symbol $\mathbf{x}$ from $\mathbf{r}_{t}^{i}$

$$
m\left(\mathbf{x} \mid \mathbf{r}_{t}^{i}, \lambda_{\ell_{1}}, \lambda_{\ell_{2}}, \ldots, \lambda_{\ell_{q_{i}}}\right)=\sum_{j=1}^{q_{i}}\left|r_{t j}^{i}-\sqrt{\lambda_{\ell_{j}} p_{j}^{i}} x_{j}\right|^{2} .
$$

It can be seen in (4) that the decoding complexity of the ML metric increases with $q_{i}$, the dimension of the signal set used. We can compute (4) more efficiently through the universal lattice decoder, commonly known as the sphere

\footnotetext{
${ }^{1}$ Multiplication by unitary matrices does not alter the statistics of a noise vector.
}

decoder [6]. The sphere decoder was shown to perform close to ML decoding with low complexity for dimensions of up to 32 . As the expected complexity of sphere decoding was shown to be polynomial (approximately cubic) [7], we note that it is practically feasible to decode the multi-dimensional symbols at the receiver.

\section{Symbol ERror Rate And OUtage Probability}

In order to capture the asymptotic performance of our system, we analyze the DMT derived in [1], where diversity order, $d$ is given by $d \equiv-\lim _{P \rightarrow \infty} \frac{\log P_{e}(P)}{\log P}$, which describes the rate at which the average error probability, $P_{e}(P)$, falls with $P$ at large $P$, for a particular target rate, $R(P)$. Multiplexing gain is defined as $r \equiv \lim _{P \rightarrow \infty} \frac{R(P)}{\log P}$, where $r$ quantifies the dependence of the target rate on the average power constraint.

If $\mathcal{E}$ is the error event, the probability of wrong detection of $\mathbf{x}_{t}^{i}$ from $\mathbf{r}_{t}^{i}$ are identical for $t=1,2$ and given by

$$
\underbrace{\operatorname{Pr}\left(x_{l} \rightarrow x_{\eta(l)}\right)}_{\text {Most probable error event }} \leq \operatorname{Pr}\left(\mathcal{E} \mid \mathbf{x}_{k}^{i}=x_{l}\right) \leq \underbrace{\sum_{x_{k} \in \mathcal{N}(l)} \operatorname{Pr}\left(x_{l} \rightarrow x_{k}\right)}_{\text {Union Bound }},
$$

where $x_{\eta(l)}$ represents the nearest neighbor of $x_{l}$ and $\mathcal{N}(l)$ represents the set of constellation points in the immediate neighborhood of $x_{l}$ in $\mathcal{O}^{\prime}$. For any $q_{i}$-dimensional QAM constellation, the maximum number of points in the immediate neighborhood of any point is $2 q_{i}$, i.e. $|\mathcal{N}(l)|=2 q_{i}$. Therefore

$$
\sum_{x_{k} \in \mathcal{N}(l)} \operatorname{Pr}\left(x_{l} \rightarrow x_{k}\right) \leq 2 q_{i} \operatorname{Pr}\left(x_{l} \rightarrow x_{\eta(l)}\right) .
$$

Since the upper bound and the lower bound in (5) are different only by a scaling factor, to calculate the DMT, we need to find the SNR exponent of the most probable error event, the lower bound in (5).

We consider $\operatorname{Pr}\left(x_{l} \rightarrow x_{k}\right)$ for any $k$ and $l$ in the immediate neighborhood of each other which is given by

$$
\operatorname{Pr}\left(x_{l} \rightarrow x_{k}\right)=\mathbb{E}\left[Q\left(\frac{\left|u_{l}-u_{k}\right|}{2}\right)\right],
$$

where $u_{\{l, k\}}=\boldsymbol{\Lambda}^{i} \overline{\mathbf{P}}^{i} x_{\{l, k\}}$ represents the received constellation point $x_{\{l, k\}}=\left[x_{\{l, k\} 1}, x_{\{l, k\} 2}, \ldots, x_{\{l, k\} q_{i}}\right]^{T}$ without any noise. We have in (7)

$$
\left|u_{l}-u_{k}\right|=\left[\sum_{j=1}^{q_{i}} \lambda_{\ell_{j}} p_{j}\left(x_{l j}-x_{k j}\right)^{2}\right]^{\frac{1}{2}} .
$$

The power allocated to each set $P_{i}$ is taken to be $P / L$. However, such a choice will not alter the DMT. Scaling the power by a constant factor, in this case $L$ will not make a difference when $P \rightarrow \infty^{2}$. Let $R_{i}=r_{i} \log P$ be the rate on the set $Q_{i}$ such that $R=r \log P=\left(\sum_{i=1}^{s} r_{i}\right) \log P$ where $R$ is the target transmission rate. For a transmission rate of $r_{i} \log P$,

\footnotetext{
${ }^{2}$ Noting that $P_{i} \leq P$ for each $i$, we could upper-bound the performance by taking $P_{i}=P$. However the DMT is unchanged from what is obtained here.
} 
there should be a total of $P^{r_{i}}$ possible points in the combined constellation of $\mathbf{x}_{1}^{i}$ and $\mathbf{x}_{2}^{i}$. As $\mathbf{x}_{1}^{i}$ and $\mathbf{x}_{2}^{i}$ are symmetric, there should be $P^{\frac{r_{i}}{2}}$ points in the constellation of either $\mathbf{x}_{1}^{i}$ or $\mathbf{x}_{2}^{i}$. Additionally, since $\mathbf{x}_{1}^{i}$ and $\mathbf{x}_{2}^{i}$ are unit energy $q_{i}$-dimensional constellations, the spacing between points is of the order of $P^{\frac{r_{i}}{2 q_{i}}}$ in each dimension. If $x_{l}$ and $x_{k}$ are $d_{j}^{l k}$ symbols apart in the $j^{\text {th }}$ dimension, the distance between them in the $j^{\text {th }}$ dimension will be of the order of $d_{j}^{l k} P^{-\frac{r_{i}}{2 q_{i}}}$. We note that as $\mathcal{O}^{\prime}$ is a rotation of the constellation $\mathcal{O}$ with $M, d_{j}^{l k} \neq 0$ for $l \neq k$, i.e. no two points have the same $j^{t h}$ coordinate. Therefore, we have in (9),

$$
\left|u_{l}-u_{k}\right|=\left[\sum_{j=1}^{q_{i}} \lambda_{\ell_{j}} p_{j}\left(d_{j}^{l k}\right)^{2} P^{-\frac{r_{i}}{q_{i}}}\right]^{\frac{1}{2}}
$$

To analyze the symbol error rate in (7) using (9), we would need the joint distribution of the ordered eigenvalues in $Q_{i}$. We define the central Wishart matrix as $\mathbf{W}=\mathbf{H H}^{\dagger}$ when $N_{r}<N_{t}$ and $\mathbf{W}=\mathbf{H}^{\dagger} \mathbf{H}$ when $N_{r}>N_{t}$.

Theorem 3.1 (Joint Distribution of Eigenvalues): An approximation to the joint distribution of eigenvalues of a central Wishart matrix $\bar{\lambda}_{\ell_{1}}^{\ell_{q}}=\left\{\lambda_{\ell_{1}}, \lambda_{\ell_{2}}, \ldots, \lambda_{\ell_{q}}\right\}$ where $\ell_{1}<\ell_{2}<\ldots<\ell_{q}$ and $\ell_{j} \in\{1,2,3, \ldots, n\}$, valid for small arguments $^{3}$ is given by

$$
\begin{aligned}
g\left(\bar{\lambda}_{\ell_{1}}^{\ell_{q}}\right)= & C\left(\prod_{i=1}^{q-1} \lambda_{\ell_{i}}^{\chi_{i}-1}\right) \lambda_{\ell_{q}}^{\chi_{q}^{\prime}-1} \\
& +o\left(\left(\prod_{i=1}^{q-1} \lambda_{\ell_{i}}^{\chi_{i}-1+\epsilon_{i}}\right) \lambda_{\ell_{q}}^{\chi_{q}^{\prime}-1+\epsilon_{q}}\right),
\end{aligned}
$$

where $C$ is the normalizing factor,

$$
\begin{gathered}
\chi_{i}=\left(\ell_{i+1}-\ell_{i}\right)\left(m+n-\ell_{i}-\ell_{i+1}+2\right), \\
\chi_{q}^{\prime}=\left(m-\ell_{q}+1\right)\left(n-\ell_{q}+1\right),
\end{gathered}
$$

and $\epsilon_{i}$ is a positive constant for $i=1,2, \ldots, q$.

Proof: We refer to [8, Lemma 2] for the approximate distribution of a single ordered eigenvalue valid for small arguments. The same result has been proved rigorously in [9]. For the sake of brevity, we present only a heuristic argument on the lines of [8]. A rigorous proof is possible with a nontrivial generalization of steps in [9, Appendix II].

The required joint distribution is exactly given by

$$
\begin{array}{r}
g\left(\bar{\lambda}_{\ell_{1}}^{\ell_{q}}\right)=\int_{\mathcal{D}_{\ell_{1}}^{+}} \int_{\mathcal{D}_{\ell_{1}, \ell_{2}}^{\times}} \int_{\mathcal{D}_{\ell_{2}, \ell_{3}}^{\times}} \ldots \int_{\mathcal{D}_{\ell_{q}}^{-}} f\left(\{\lambda\}_{i=1,2, \ldots, n}\right) \\
d \lambda_{\ell_{\mathbf{1}}}^{+} d \lambda_{\ell_{\mathbf{1}}, \ell_{\mathbf{2}}}^{\times} d \lambda_{\ell_{\mathbf{2}}, \ell_{\mathbf{3}}}^{\times} \ldots d \lambda_{\ell_{\mathbf{q}-1}, \ell_{\mathbf{q}}}^{\times} d \lambda_{\ell_{\mathbf{q}}}^{-},
\end{array}
$$

where

$$
f\left(\lambda_{1}, \lambda_{2}, \ldots, \lambda_{n}\right)=K_{m, n}^{-1} e^{-\sum_{i} \lambda_{i}} \prod_{i} \lambda_{i}^{m-n} \prod_{i<j}\left(\lambda_{i}-\lambda_{j}\right)^{2}
$$

\footnotetext{
${ }^{3}$ We write a function $a(x)$ as $o(x)$ if $\lim _{x \rightarrow 0} a(x) / x=0$.
}

is the complete joint distribution of all the eigenvalues for the Rayleigh channel. Also

$$
\begin{gathered}
\lambda_{\ell_{\mathbf{1}}}^{+}=\left\{\lambda_{1}, \ldots, \lambda_{\ell_{1}-1}\right\}, \lambda_{\ell_{\mathbf{q}}}^{-}=\left\{\lambda_{\ell_{q+1}}, \ldots, \lambda_{n}\right\}, \\
\lambda_{\ell_{\mathbf{i}}, \ell_{\mathbf{i}+\mathbf{1}}}^{\times}=\left\{\lambda_{\ell_{i}+1}, \ldots, \lambda_{\ell_{i+1}-1}\right\}
\end{gathered}
$$

and their respective domains

$$
\begin{gathered}
\mathcal{D}_{\ell_{1}}^{+}=\left\{\lambda_{\ell_{1}} \leq \lambda_{\ell_{1}-1} \leq \cdots \leq \lambda_{1}<\infty\right\}, \\
\mathcal{D}_{\ell_{q}}^{-}=\left\{0<\lambda_{n} \leq \lambda_{n-1} \leq \cdots \leq \lambda_{\ell_{q}+1} \leq \lambda_{\ell_{q}}\right\}, \\
\mathcal{D}_{\ell_{i}, \ell_{i+1}}^{\times}=\left\{\lambda_{\ell_{i}} \leq \lambda_{\ell_{i}+1} \leq \cdots \leq \lambda_{\ell_{i+1}-1} \leq \lambda_{\ell_{i+1}}\right\}
\end{gathered}
$$

for $i=1,2, \ldots, q-1$.

The integration over $\mathcal{D}_{\ell_{1}}^{+}$results in a constant and is not a function of $\lambda_{\ell_{1}}$ for small $\lambda_{\ell_{1}}$. We turn to integration over $\mathcal{D}_{\ell_{i}, \ell_{i+1}}^{\times}$for $i=q-1, q-2, \ldots, 1$. As the complete joint distribution of (13), $f(\cdot) \rightarrow 0$, whenever $\lambda_{k} \rightarrow \lambda_{k+1}$, we assume that the eigenvalues are separated widely enough and approximate $\left(\lambda_{k}-\lambda_{j}\right)^{2}$ in (13) with $\lambda_{k}^{2}$ for all $k<j$ without loss of accuracy for further calculations. For each $\lambda_{k} \in \lambda_{\ell_{\mathbf{i}}, \ell_{\mathbf{i}+1}}^{\times}$, there are $n-k$ possible factors $\left(\lambda_{k}-\lambda_{j}\right)^{2} \approx \lambda_{k}^{2}$ along with $\lambda_{k}^{m-n}$. Therefore, $\chi_{i}-1$, the exponent of $\lambda_{\ell_{i}}$ is

$$
\begin{aligned}
\chi_{i}-1 & =\sum_{k=\ell_{i}}^{\ell_{i+1}-1}\{(m-n)+2(n-k)+1\}-1 \\
& =\left(\ell_{i+1}-\ell_{i}\right)\left(m+n-\ell_{i+1}-\ell_{i}+2\right)-1 .
\end{aligned}
$$

Similar arguments hold for integration over $\mathcal{D}_{\ell_{q}}^{-}$to obtain

$$
\chi_{q}^{\prime}-1=\left(m-\ell_{q}+1\right)\left(n-\ell_{q}+1\right)-1,
$$

which can be checked by substituting $\ell_{i+1}=n+1$ in (19).

We verify that the marginal distributions obtained from the joint distribution derived here coincide with results in [8, Lemma 2] and [9, Theorem 1].

$$
\begin{aligned}
g\left(\bar{\lambda}_{\ell_{1}}^{\ell_{q-1}}\right) & =\int_{\lambda_{\ell_{q}}=0}^{\lambda_{\ell_{q-1}}} g\left(\bar{\lambda}_{\ell_{1}}^{\ell_{q}}\right) d \lambda_{\ell_{q}} \\
& =C \lambda_{\ell_{1}}^{\chi_{1}-1} \lambda_{\ell_{1}}^{\chi_{2}-1}, \ldots, \lambda_{\ell_{q-1}}^{\chi_{q-1}+\chi_{q}^{\prime}-1} \\
& \stackrel{(a)}{=} C \lambda_{\ell_{1}}^{\chi_{1}-1} \lambda_{\ell_{1}}^{\chi_{2}-1}, \ldots, \lambda_{\ell_{q-1}}^{\chi_{q-1}^{\prime}-1},
\end{aligned}
$$

where in (a) we make use of a property which is easily verified.

$$
\chi_{i-1}+\chi_{i}^{\prime}-1=\chi_{i-1}^{\prime}-1
$$

for $i=2, \ldots, q$. Proceeding in the same manner we arrive at the marginal distribution $g\left(\lambda_{\ell_{1}}\right)=C \lambda_{\ell_{1}}^{\chi_{1}^{\prime}}$ which corresponds to the results in [8, Lemma 2] and [9, Theorem 1].

We now proceed to state the DMT for the set $Q_{i}$ in our second main result. The proof is supplied in the appendix.

Theorem 3.2 (DMT within a set): The DMT for the average error probability on the set $Q_{i}$ using $q_{i}$-dimensional symbols as given in (2) is

$$
d_{i}\left(r_{i}\right)=\left(m-\mu_{i}+1\right)\left(n-\mu_{i}+1\right)\left(1-\frac{r_{i}}{q_{i}}\right),
$$

where $\mu_{i}$ is the strongest eigenchannel in $Q_{i}$ and $q_{i}=\left|Q_{i}\right|$. 
From (22), we see that the DMT for $Q_{i}$ depends only on the strongest eigenchannel in $Q_{i}$. Therefore, we can infer that the effect of the weaker eigenchannels is offset by the method of co-ordinate interleaving. The best performance can be expected when we interleave coordinates of $n$ dimensional symbols across the $n$ eigenchannels, i.e. when we have only one set. From Theorem 3.2, we see that the DMT for interleaving coordinates across all the eigenchannels is given by a straight line joining the points corresponding to maximum diversity $(0, m n)$ and maximum multiplexing gain $(n, 0)$. If we wish to use constellations with smaller dimensions to reduce decoding complexity, the following result describes appropriate partitioning of eigenchannels into sets.

Theorem 3.3 (System DMT): For the co-ordinate interleaved system with the partition of eigenchannels $\left\{Q_{i}\right\}_{i=1}^{s}$, the optimal DMT $d_{D}^{*}(r)$ is given by a piece-wise linear curve joining the points

$$
\left(\rho_{k},\left(m-\mu_{k+1}+1\right)\left(n-\mu_{k+1}+1\right)\right) \quad k=0,1,2, \ldots, s
$$

where

$$
\rho_{k}= \begin{cases}0 & k=0 \\ \sum_{j=1}^{k} q_{j}\left(1-\frac{\left(m-\mu_{k+1}+1\right)\left(n-\mu_{k+1}+1\right)}{\left(m-\mu_{j}+1\right)\left(n-\mu_{j}+1\right)}\right) & k>0,\end{cases}
$$

$q_{j}=\left|Q_{j}\right|, \mu_{i}$ is the strongest eigenchannel in $Q_{i}$ for $i=$ $1,2, \ldots, s$ and $\mu_{s+1}=n+1$. For each $r>0$, the rates allocated to $\left\{Q_{i}\right\}$ are given by

$$
r_{i}= \begin{cases}q_{i}\left(1-\frac{d_{D}^{*}(r)}{\left(m-\mu_{i}+1\right)\left(n-\mu_{i}+1\right)}\right) & 1 \leq i \leq \min _{\rho_{L} \geq r} L \\ 0 & i>\min _{\rho_{L} \geq r} L\end{cases}
$$

Proof: The problem of finding the optimum DMT reduces to an optimization problem of allocating rates to the different sets $Q_{i}$ depending on their relative strengths. Let $\mu_{s+1}=n+1$ and

$$
\begin{gathered}
c(k)=\left(m-\mu_{k}+1\right)\left(n-\mu_{k}+1\right) \quad k=1,2, \ldots, s \\
D(L, r)=c(i)\left(1-\frac{r_{i}}{q_{i}}\right) \quad i=1,2, \ldots, L \quad L \leq s .
\end{gathered}
$$

We have to find the optimum DMT $d_{D}^{*}(r)=\max _{L} D(L, r)$ subject to the constraint $\sum_{i=1}^{L} r_{i}=r$. Solving the problem, we get the transition points mentioned in Theorem 3.3 when $D\left(i, \rho_{i}\right)=D\left(i+1, \rho_{i}\right)$ for $i=1,2, \ldots, s-1$. At the transition point $\left(\rho_{k}, c(k+1)\right)$, the diversity $d_{D}^{*}(r)$ is equal to the best diversity of the strongest eigenchannel in set $Q_{k+1}$.

Remark 3.1: From Theorem 3.3, it is clear that the optimum patitioning of eigenchannels to $\left\{Q_{i}\right\}$ would imply $\mu_{i}=i$ i.e. allocate the $s$ largest eigenvalues separately to the $s$ sets.

Remark 3.2: We turn the tradeoff around and consider the rate-diversity trade-off $r_{D}^{*}(d)$ for our method and $r_{M}^{*}(d)$ for multiple beamforming [2]. Let us also assume the partitioning as in Remark 3.1, i.e. $\mu_{i}=i$ for $i=1,2, \ldots, s$. In Theorem 3.3 , if we substitute equal $q_{i}=q$, it is not difficult to show that at points where diversity $d(r)=(m-i+1)(n-i+1)$ for $i=1,2, \ldots, s$, the ratio of the rates $r_{D}^{*}(d) / r_{M}^{*}(d)=$ $q$, the constellation dimension employed. Even with a QAM

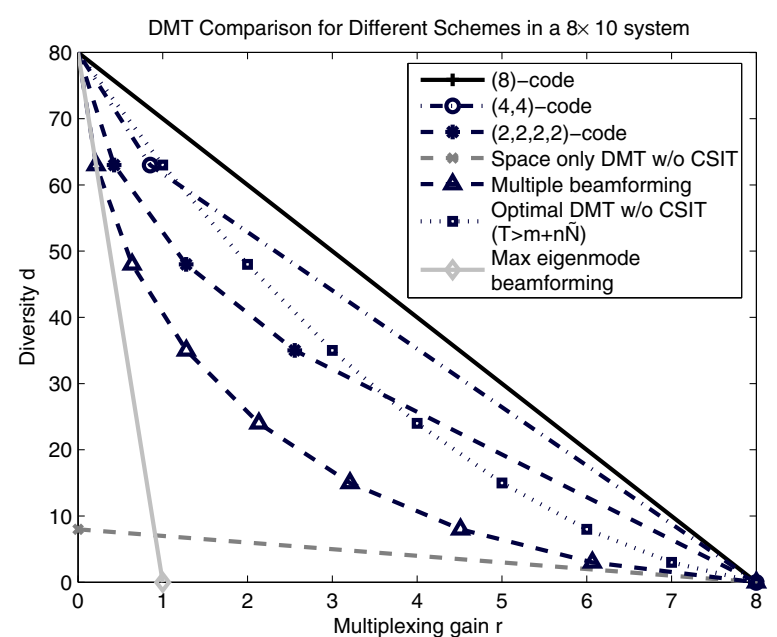

Fig. 1. DMT Comparison for a $8 \times 10$ system

constellation employed in our system we get a gain of 2 over multiple beamforming. As we increase the dimension of the signal space, we can extract corresponding gains in spatial multiplexing at a given reliability.

\section{REPRESENTATIVE RESUlTS FOR A $8 \times 10$ MIMO SYSTEM}

In Figure 1, we plot the DMT for our scheme applied to an $8 \times 10$ system with different partitions and compare it with other curves. In the $(8)$-code, we use 8-dimensional codes to interleave across all 8 eigenchannels. In the $(4,4)$-code we have two sets $Q_{1}$ and $Q_{2}$ containing $\lambda_{1}$ and $\lambda_{2}$ respectively. Irrespective of how $\lambda_{3}, \ldots, \lambda_{8}$ are allocated to $Q_{1}$ and $Q_{2}$ the DMT remains unchanged as explained before. However, the coding gains might depend on the exact partition used. Similarly, in the $(2,2,2,2)$-code, we have $Q_{1}, Q_{2}, Q_{3}$ and $Q_{4}$ containing $\lambda_{1}, \lambda_{1}, \lambda_{3}$ and $\lambda_{4}$ respectively.

When compared with multiple beamforming [2], we notice substantial gains with all partitions. In contrast to [2] where the method suffers from the outage of the weaker eigenchannels, in our method, we harness signal space diversity through interleaving symbols across the eigenchannels. At high multiplexing gains, the performances of the (8)-code and $(4,4)$-code are quite close and significantly higher than other curves. In a practical system, we might opt for the lower complexity $(4,4)$-code and still get substantial gains in performance. We also plot in Figure 1 the fundamental DMT's for space-only coding (coherence length $T=1$ ) and space time coding (coherence length $T \geq m+n-1$ ) with CSIR only [1]. Since our method is essentially a space-only code, comparison with the former would be appropriate.

\section{CONCLUSION}

In the current work, we proposed a general model for coordinate interleaved spatial multiplexing that seeks to leverage the strong eigenchannels of a MIMO system to offset the weaker ones. Substantial gains over other SVD transmission 
schemes were observed particularly in the high multiplexing gain regime. We also note that there is considerable flexibility in our scheme with respect to the choice of the constellation dimensions. In future work, we would add temporal power [10] and rate control [11] to our model. We would also examine the effect of imperfect channel state information on the performance of our method.

\section{APPENDIX}

Proof: [of Theorem 3.2] Let the joint distribution $g\left(\bar{\lambda}_{\ell_{1}}^{\ell_{q_{i}}}\right)$ derived in Theorem 3.1, be valid for $\lambda_{\ell_{1}}<\Delta$ for some $\Delta>0$. With a slight abuse of notation from Theorem 3.1, we denote the domain of integration $0 \leq \lambda_{\ell_{q_{i}}} \leq \lambda_{\ell_{q_{i}-1}} \leq$ $\ldots \lambda_{\ell_{1}}$ and $B_{1} \leq \lambda_{\ell_{1}} \leq B_{2}$ by $D_{B_{1}}^{B_{2}}$.

$$
\begin{aligned}
\operatorname{Pr}\left(x_{l} \rightarrow x_{k}\right)= & \underbrace{\int_{D_{0}^{\Delta}}\left[Q\left(\frac{\left|u_{l}-u_{k}\right|}{2}\right)\right] g\left(\bar{\lambda}_{\ell_{1}}^{\ell_{q_{i}}}\right) d \bar{\lambda}_{\ell_{1}}^{\ell_{q_{i}}}}_{\text {(I) }} \\
& +\underbrace{\int_{D_{\Delta}^{\infty}}\left[Q\left(\frac{\left|u_{l}-u_{k}\right|}{2}\right)\right] f\left(\bar{\lambda}_{\ell_{1}}^{\ell_{q_{i}}}\right) d \bar{\lambda}_{\ell_{1}}^{\ell_{q_{i}}}}_{\text {(II) }} .
\end{aligned}
$$

Terms (II) is $O\left(\frac{1}{{ }_{P}^{\left(1-\frac{r_{i}}{q_{i}}\right) m n}}\right)$ using the bound $Q(x) \leq e^{-\frac{x^{2}}{2}}$.

Using the result $Q(x)=\frac{1}{\pi} \int_{0}^{\frac{\pi}{2}} e^{-\frac{x^{2}}{2 \sin ^{2} t}} d t$ and changing the order of integration, term (I) is expanded as

$$
\begin{aligned}
& \int_{D_{0}^{\Delta}}\left[Q\left(\frac{\left|u_{l}-u_{k}\right|}{2}\right)\right] g\left(\bar{\lambda}_{\ell_{1}}^{\ell_{q_{i}}}\right) d \bar{\lambda}_{\ell_{1}}^{\ell_{q_{i}}} \\
& =\frac{1}{\pi} \int_{0}^{\frac{\pi}{2}} \underbrace{\int_{D_{0}^{\Delta}} e^{\left[-\frac{{ }_{P}\left(-\frac{r_{i}}{q_{i}}\right)_{\sum_{j=1}^{q_{i} p_{j} \lambda_{j}}\left(d_{j}^{l k}\right)^{2}}}{\sin ^{2} t}\right]} g\left(\bar{\lambda}_{\ell_{1} q_{i}}\right) d \bar{\lambda}_{\ell_{1}}^{\ell_{q_{i}}}}_{I} d t .
\end{aligned}
$$

We obtain the lower bound for the DMT of the system, $d_{i, L}\left(r_{i}\right)$ by initially assuming uniform power allocation for $\left\{p_{k}\right\}_{i=1}^{q_{i}}, \underline{p_{k}}=\frac{1}{q_{i}} P_{i}=\frac{P}{L q_{i}}$ in (28). We concentrate on the inner integral $I$. In the expression for $g\left(\bar{\lambda}_{\ell_{1}}^{\ell_{q_{i}}}\right)$, we omit the $o\left(\left(\prod_{i=1}^{q_{i}-1} \lambda_{\ell_{i}}^{\chi_{i}-1+\epsilon_{i}}\right) \lambda_{\ell_{q_{i}}}^{\chi_{q_{i}}^{\prime}-1+\epsilon_{q_{i}}}\right)$ term as it does not influence the calculation of DMT and write $I$ as

$$
\begin{aligned}
& =C \int_{0}^{\Delta} \int_{0}^{\lambda_{\ell_{1}}} \cdots \int_{0}^{\lambda_{\ell_{q_{i}-1}}} \prod_{j=1}^{q_{i}-1} e^{\left[-\frac{{ }^{\left(1-\frac{r_{i}}{q_{i}}\right)}{ }_{\lambda_{\ell_{j}}\left(d_{j}^{l k}\right)^{2}}}{L q_{i} \sin ^{2} t}\right]} \lambda_{\ell_{j}}^{\chi_{j}-1}
\end{aligned}
$$

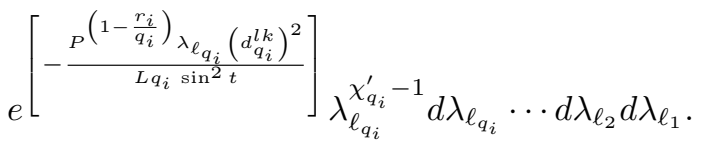

We notice that the inner integrals are separable and lower incomplete gamma functions which are of the form

$$
\int_{0}^{\lambda_{\ell_{i}}} e^{-\Gamma \lambda_{\ell_{i+1}}} \lambda_{\ell_{i+1}}^{a-1} d \lambda_{\ell_{i+1}}=\frac{(a-1) !}{\Gamma^{a}}\left[1-e^{-\Gamma \lambda_{\ell_{i}}} \sum_{v=0}^{a-1} \frac{\lambda_{\ell_{i}}^{v}}{v !}\right],
$$

where $\Gamma \propto \frac{P^{\left(1-\frac{r_{i}}{L q_{i}}\right)}\left(d_{i+1}^{l k}\right)^{2}}{q_{i} \sin ^{2} t}$ and $a$ is an integer. We now begin with the innermost integral in (29) and proceed outwards, taking $i=q_{i}-1, q_{i}-2, \ldots, 1$ in (30). After a few manipulations and utilizing the property in (21), we get

$$
I=C^{\prime}\left(\frac{P^{\left(1-\frac{r_{i}}{q_{i}}\right)}}{\sin ^{2} t}\right)^{-\chi_{1}^{\prime}}+o\left(\left(\frac{P^{\left(1-\frac{r_{i}}{q_{i}}\right)}}{\sin ^{2} t}\right)^{-\left(\chi_{1}^{\prime}+\epsilon^{\prime}\right)}\right)
$$

where $C^{\prime}$ is a new constant and $\epsilon^{\prime}>0$. Substituting $I$ from (31) back in (28), we have

$\operatorname{Pr}\left(x_{l} \rightarrow x_{k}\right)=C^{\prime \prime}\left(P^{\left(1-\frac{r_{i}}{q_{i}}\right)}\right)^{-\chi_{1}^{\prime}}+o\left(P^{-\left(1-\frac{r_{i}}{q_{i}}\right)\left(\chi_{1}^{\prime}+\epsilon^{\prime}\right)}\right)$,

where $C^{\prime \prime}=\frac{C^{\prime}}{\pi} \int_{0}^{\frac{\pi}{2}}(\sin t)^{2 \chi_{1}^{\prime}} d t$ is yet another constant. From (32) and (5), we have the lower bound to the DMT as

$$
d_{i, L}\left(r_{i}\right)=\left(m-\ell_{1}+1\right)\left(n-\ell_{1}+1\right)\left(1-\frac{r_{i}}{q_{i}}\right) .
$$

We now obtain an upper bound for the DMT of the system, $d_{i, U}\left(r_{i}\right)$ by setting choosing $\left\{p_{k}\right\}_{i=1}^{q_{i}}$ to be $\overline{p_{k}}=P_{i}=\frac{P}{q_{i}}$. Since any choice $\sum_{k=1}^{q_{i}} p_{k} \leq P_{i}$, the DMT obtained with $\overline{p_{k}}$ is an upper bound to the performance. Noting that $\overline{p_{k}}$ is only a scaled version of $p_{k}$, we see that the upper bound for the DMT coincides with the lower bound $d_{i, U}\left(r_{i}\right)=d_{i, L}\left(r_{i}\right)$.

\section{REFERENCES}

[1] L. Zheng and D. N. C. Tse, "Diversity and Multiplexing: A Fundamental Tradeoff in Multiple-Antenna Channels," IEEE Trans. Info. Theory, vol. 49, no. 5, pp. 1073-1096, May 2003.

[2] L. Garcia-Ordonez, A. Pages-Zamora, and J. R. Fonollosa, "Diversity and Multiplexing Tradeoff of Multiple Beamforming in MIMO Channels,' in IEEE Int. Symp. Inform. Theory, ISIT'05, Adelaide, Australia, 2005.

[3] K. V. Srinivas, J. K. Milleth, R. D. Koilpillai, and K. Giridhar, "Coordinate Interleaved Spatial Multiplexing with Channel Knowledge at Transmitter and Receiver," in Proc. IEEE Int. Conf. Acoust., Speech, Signal Process., ICASSP, Toulouse, France, 2006.

[4] K. Boulle and J. C. Belfiore, "Modulation schemes designed for the rayleigh channel," in Proc. Conference on Information Sciences and Systems (CISS), Princeton, NJ, Mar. 1992, pp. 288-293.

[5] J. Boutros and E. Viterbo, "Signal space diversity: a power and bandwidth efficient diversity technique for the fading channel," IEEE Trans. Info. Theory, vol. 44, no. 4, pp. 1453-1467, July 1998.

[6] E. Viterbo and J. Boutros, "A Universal Lattice Code Decoder for Fading Channels," IEEE Trans. Info. Theory, vol. 45, no. 5, pp. 1639-1642, July 1999.

[7] B. Hassibi and H. Vikalo, "On the Sphere-Decoding Algorithm-Expected Complexity," IEEE Trans. Signal Proc., vol. 53, no. 8, pp. 2806-2818, August 2005.

[8] A. Khoshnevis and A. Sabharwal, "On the Asymptotic Performance of Multiple Channels with Quantized Feedback," submitted to IEEE Trans. Wireless Comm., 2007.

[9] L. Garcia-Ordonez, D. P. Palomar, A. Pages-Zamora, and J. R. Fonollosa, "High-SNR Analytical Performance of Spatial Multiplexing MIMO Systems With CSI," IEEE Trans. Signal Proc., vol. 55, no. 11, pp. 54475463, November 2007.

[10] C. Steger and A. Sabharwal, "Single-Input Two-Way SIMO Channel: Diversity-Multiplexing Tradeoff with Two-Way Training," accepted for publication in IEEE Trans. Wireless Comm., June 2007.

[11] A. W. C. Lim and V. K. N. Lau, "On the Fundamental Tradeoff of Spatial Diversity and Spatial Multiplexing of MIMO Links with Imperfect CSIT,' in IEEE Int. Symp. Inform. Theory, ISIT'06, Seattle, USA, July 9-14 2006, pp. 2704-2708. 